\title{
Finger Vein Recognition using Two Parallel Enhancement Approachs based Fuzzy Histogram Equalization
}

\author{
Khamis A.Zidan ${ }^{1}$, Shereen.S.Jumaa ${ }^{2}$ \\ ${ }^{1}$ Al-Iraqia University ,Iraq \\ 2 Al-Nahrain University, College of Information Engineering,Iraq
}

\begin{tabular}{ll} 
Article Info & ABSTRACT \\
\cline { 2 - 3 } Received Dec 31,2018 & $\begin{array}{l}\text { This paper evaluates a set of enhancement stages for finger vein } \\
\text { enhancement that not only has low computational complexity but also } \\
\text { high distinguishing power. This proposed set of enhancement stages is } \\
\text { centered around fuzzy histogram equalization. Two sets of evaluation } \\
\text { are carried out: one with the proposed approach and one with another }\end{array}$ \\
\hline Keyword: & $\begin{array}{l}\text { unique approach that was formulated by rearranging and cropping } \\
\text { down the preprocessing steps of the original proposed approach. To }\end{array}$ \\
Finger vein & $\begin{array}{l}\text { extract features, a combination of Hierarchical Centroid and Histogram } \\
\text { of Gradients was used. Both enhancement stages were evaluated with }\end{array}$ \\
Histogram equalization & K Nearest Neighbor and Deep Neural Networks using 6 fold stratified \\
Ceep Neural Network & cross validation. Results showed improvement as compared to three \\
latest benchmarks in this field that used 6-fold validation.
\end{tabular}

\section{Corresponding Author:}

First Author,

Al-Iraqia University ,Iraq

Email: khamis_zidan@yahoo.com

\section{Introduction}

In the field of smart recognition, identification of individual's unique attributes is now a global security concern[1][2]. A number of algorithms have been developed in the past few years but improvements can still be incorporated for more efficient biometric recognition algorithms. Biometric recognition refers to automation in recognizing an individual's unique identity on the basis of his anatomic/behavioral characteristics. Anatomic features include fingerprint, palm print, hand veins, finger veins, palm veins, foot vein, iris, gait and DNA recognition. Based on these features, a number of biometric techniques has been already presented [3]. Following are the two categories of biometric recognition approaches:

(i) Features that are extrinsic i.e. palm print, iris, fingerprint and face

(ii) Features that are intrinsic i.e. palm vein, hand vein, and finger vein [4].

Extrinsic features are more clear and exposed as compared to intrinsic features and also have more unfavorable aspects. For example, during the extraction of iris features, the surface of retina may be affected by transmitting high intensity light [5]. Similarly, distortion can occur in face identification due to various factors such as brightness, variance, and pose [6].

In 2002, [7] introduced finger vein recognition technique [8]. The typical framework of the biometric finger vein recognition (FVR) system is presented in (see figure 1). 


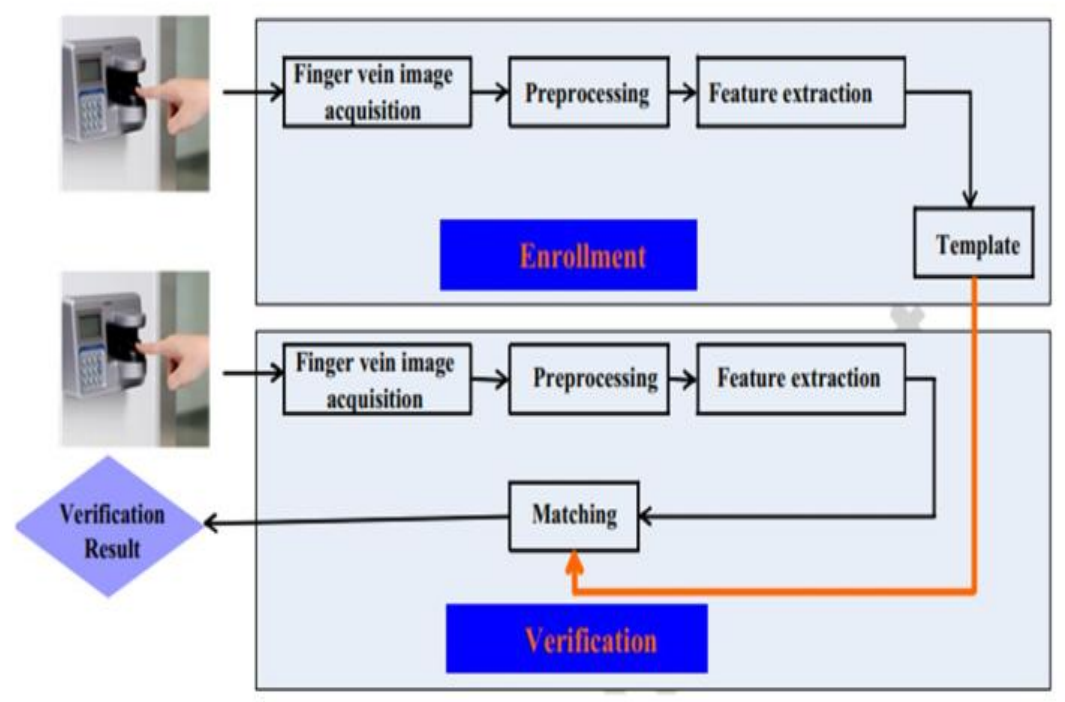

Figure 1.Typical framework for finger vein recognition [9]

Finger vein comes under the category of intrinsic features, and is therefore hard to replicate and alter. In a trans-illumination manner, near-infrared (NIR) light $(700-900 \mathrm{~nm})$ is used to capture finger veins [10]. For personal verification, systems based on veins generally use diverse anatomic features like finger vein, hand vein, foot vein or palm vein. The most recommended is finger vein because it has the smallest imaging tool and has larger number of veins as compared to palm and hand [6]. Additionally, even for the identical twins, vein pattern for every finger is unique and exists only for humans as long as they are alive [11]. More importantly, during lifetime, a finger vein pattern does not alter [5].

Finger vein recognition is viewed as one of the most challenging tasks because of low image contrast, unevenillumination and variations in temperature. Finger vein identification systems are also secure against spoof attacks [5] [10] [12] [13]. Therefore, FVR still need more fast and efficient methods.

\section{Database}

To evaluate our algorithm, we chose SDUMLA-HMT database(see figure 2). It is a fact [14] [15] that SDUMLA-HMT database is the most tough finger vein database for pre-processing. Following are the reasons for this:

1. Image quality is poor [16].

2. Overall image size is larger than the finger area [16].

3. Images vary in rotation, translation and shift [17].

4. An uncontrolled image capturing method was deployed [14]
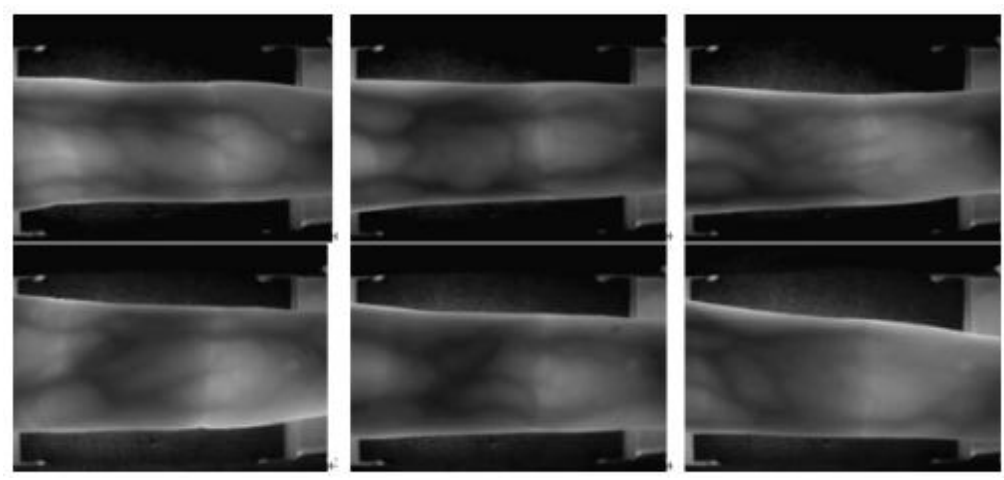

Figure 2. SDUMLA-HMT database- sample data

In compilation of images, a number of people who take part is one hundred and six (106). The format of images of vein is .bmp. Of each person, images of both two hands were taken. Data of three fingers were 
collected i.e. index, middle and ring fingers for each hand [18] and for each finger, 6 samples were obtained. For this purpose(see table 1), Joint Lab for Intelligent Computing and Intelligent Systems of Wuhan University made a device that was used.

Table 1. SDUMLA database at a glance

\begin{tabular}{|l|l|}
\hline Parameter & Information \\
\hline Total persons & 106 \\
\hline Hands per person & 2 \\
\hline Fingers per hand & 3 \\
\hline Images per finger & 6 \\
\hline Image resolution & $320 \times 240$ \\
\hline Total size of database & $0.85 \mathrm{~Gb}$ \\
\hline
\end{tabular}

\section{Methodology}

This section details the methodology followed for preprocessing of finger vein images prior to recognition stage.

\subsection{Preprocessing and enhancement of images}

Following are the steps followed:

\subsubsection{Detection of finger}

Infrared radiation is thrown on finger to capture the images. Deoxygenated hemoglobin flows in the veins, due to this infrared radiations are absorbed by the veins and so that portion is more darker than the tissue that is in the surrounding [19].

In the first step, the region of finger is detected and crop that region from the image. To do this localization, the filter that is used is mask filter (see figure 3) which follows the procedure as [20]. After the normalization of finger region, extraction of texture was done. As the finger region has lighter color than the rest of the image, on this basis a mask was created. By using a mask filter, localization of finger region was done following the method of [20].

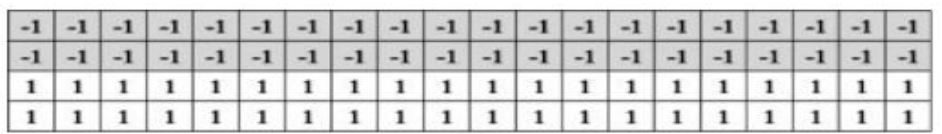

(a)

\begin{tabular}{|c|c|c|c|c|c|c|c|c|c|c|c|c|c|c|c|c|c|c|c|}
\hline 1 & 1 & 1 & 1 & 1 & 1 & 1 & 1 & 1 & 1 & 1 & 1 & 1 & 1 & 1 & 1 & 1 & 1 & 1 & 1 \\
\hline 1 & 1 & 1 & 1 & 1 & 1 & 1 & 1 & 1 & 1 & 1 & 1 & 1 & 1 & 1 & 1 & 1 & 1 & 1 & 1 \\
\hline-1 & -1 & -1 & -1 & -1 & -1 & -1 & -1 & -1 & -1 & -1 & -1 & -1 & -1 & -1 & -1 & -1 & -1 & -1 & -1 \\
\hline-1 & -1 & -1 & -1 & -1 & -1 & -1 & -1 & -1 & -1 & -1 & -1 & -1 & -1 & -1 & -1 & -1 & -1 & -1 & -1 \\
\hline
\end{tabular}

(b)

Figure 3. Masks for localizing the finer regions of the captured images. (a) Mask for detecting the upper region of the finger. (b) Mask for detecting the lower region of the finger.

Boundary of the finger is detected by computation of the masking value in y dimension for each value along the $\mathrm{x}$ dimension. This masking value has a point of maximization which is actually the boundary of the finger in y direction [21].The database image is then cropped by keeping the portion of only those rows which are fully white on the correspoinding mask(see figure 4).

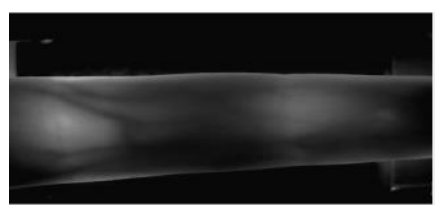

(a)

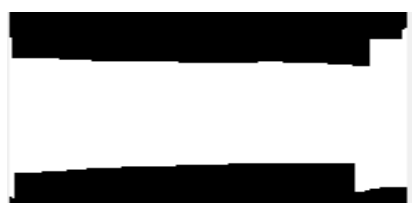

(b)

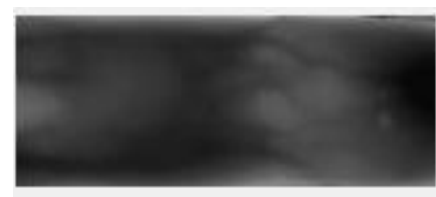

(c)

Figure 4.(a)Database image,(b)Mask obtained ,(c)Cropped finger. 


\subsubsection{Gamma correction}

Using Gamma correction, brightness of the cropped finger is enhanced [22] [23]. Manipulating the pixels nonlinearly, enhances the dynamic range of pixel intensities. It is obtained by minimizing the homogeneity of cooccurrence matrix [24] [25]. It raises the quality of image effectively by steering the average brightness of an image in the direction desired.

In proposed algorithm, it was observed that if gamma parameter is kept to be 2, it gives most optimum results (see figure 5):

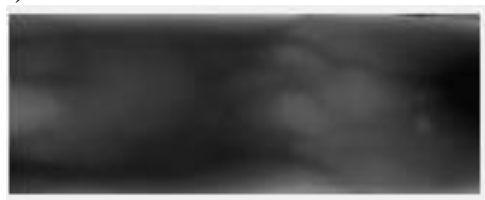

(a)

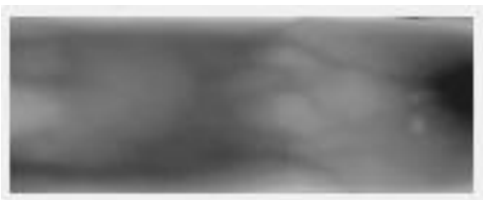

(b)

Figure 5.(a) Before Gamma correction , (b) After Gamma correction

\subsubsection{Vein sharpening}

To expose the vascular structure more clearly, to improve the quality of image, algorithm used is known as unsharp masking algorithm. The first step of the process is to make a blur and an unsharp type of image. To find out the edges, the unsharp image is subtracted from the original image. To get more sharper image, there is a particular increase in contrast along with the edges that are detected [26] [27].

As the intensity of pixels vary in range, this is done to get better contrast results between ranges [28]. It shows the hidden visual details that a human eye can never see [29]. The quality of the image is improved and shows the details that were hidden by the image sharpening process.

A Gaussian filter is used to create the unsharp mask. This filter is used particularly for finger vein gamma enhanced image. To get this filter, convolution operation of the image with kernel mask has to be done. The parameter is the standard deviation of Gaussian low pass filter which is used during sharpening of the image and so increase the image quality around the edges. During experiments, the value of this parameter is set to 4. If a value is larger, it effects the larger area and vice versa. During experimentation, sharpening strength is also set to 4 .

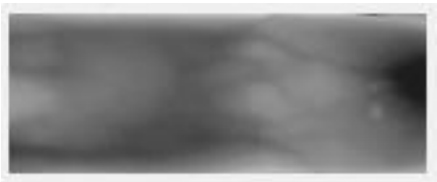

(a)

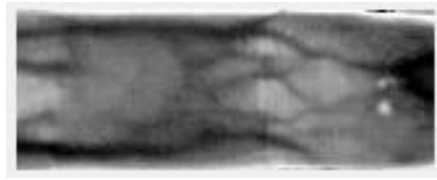

(b)

Figure 6.(a) Before Sharpening,(b) After Sharpening

\subsubsection{Double Histogram Equalization}

To tune the intensity distribution which those pixel values contain, the step is to perform histogram equalization. Combining the power of fuzzy histogram equalization with cumulative histogram equalization, and so prefer double histogram equalization. Histogram equalization is used to evenly spread pixel intensity histogram to raise the range of pixels which is dynamic and so raises the contrast.

Cumulative histogram equalization was done before Fuzzy histogram equalization.

\section{a) Cumulative histogram equalization}

Following are the steps to perform for Cumulative histogram equalization of an image [30]:

1. Image histogram is obtained first.

2. Histogram of cumulative distribution function is obtained.

3. For each gray value of original image, a new corresponding value is found by histogram equalization formula.

If:

total number of pixels $=\mathrm{N}$

total number of possible intensity levels $=\mathrm{L}$

then [31],

$$
S_{k}=\frac{L}{N} * C_{R}(k)-1
$$


In above equation:

' $\mathrm{k}$ ' is an original intensity integer value such that $\mathrm{k}$ lies between 0 and $\mathrm{L}-1$.

Sk is the value of intensity that is mapped.

1. Now from the original image, its each grey value is replaced by its respective new value that is computed from the above. Vein sharpening gives image and its corresponding histogram (see figure 7)

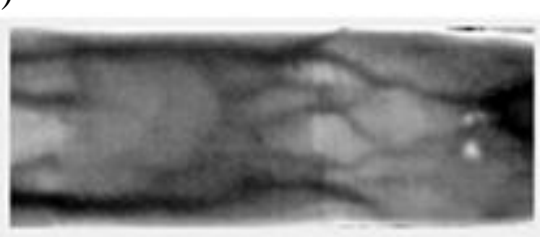

(a)

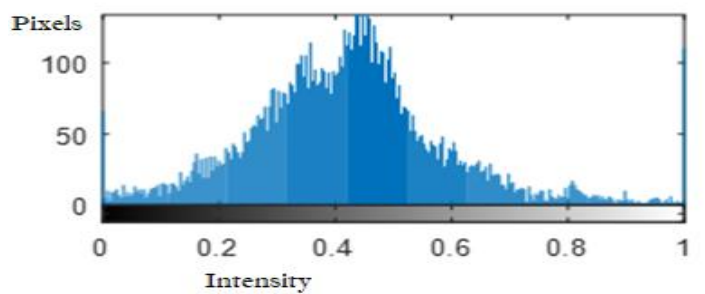

(b)

Figure 7.(a) Image before Histogram equalization , (b)Image histogram

The image as shown in figure 8(a) shows the results after cumulative histogram equalization. In comparison to figure $8(\mathrm{~b})$ it is flat.

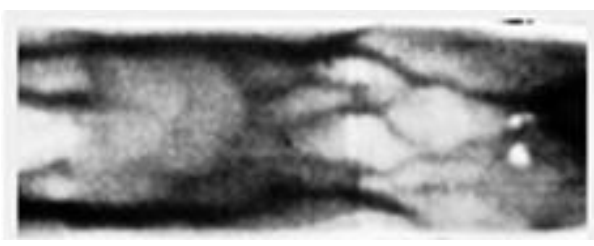

(a)

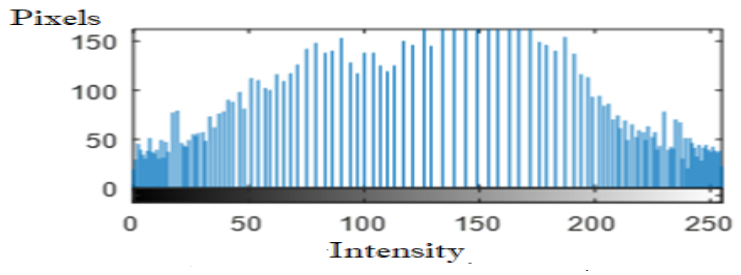

(b)

Figure 8.(a)Image After Cumulative Histogram equalization,(b) Image Histogram

A figure 8(b) shows the distribution of histogram is more even than the 7(b) but there is a congestion of pixel intensities at the edges of the graph that are horizontal. To counter this effect, fuzzy histogram equalization was performed next.

\section{b) Fuzzy histogram equalization}

Dynamic fuzzy histogram equalization does not affect the brightness [32] and it treats the histogram image in a way that mapping of peaks does not occur again. The values of histogram are distributed again in a uniform way between valley like areas of two consecutive peaks(see figure 9). Following are the steps [32]:

1. Computation of a histogram

2. Partitioning of a histogram

3. Dynamic Histogram Equalization of Partitions using fuzzy statistics

4. Normalization of image brightness if needed

A fuzzy histogram is a sequence of real numbers

$h(i), i \in\{0,1, \ldots, L-1\}$ where $h(i)$ is the frequency of occurrence of gray levels that are "around $\mathrm{i}$ ". Each gray value $\mathrm{I}(\mathrm{x}, \mathrm{y})$ is considered a fuzzy number $\mathrm{I} \sim(\mathrm{x}, \mathrm{y})$. Fuzzy histogram is then computed as:

$\mathrm{h}(\mathrm{i}) \leftarrow \mathrm{h}(\mathrm{i})+\sum_{x} \sum_{y} \mu_{I(x, y) i}, \mathrm{k} \in[a, b]$

Here $\mu_{I(x, y) i}$ is the triangular fuzzy membership function given by:

$$
\mu_{I(x, y) i}=\max \left[0,1-\frac{|I(x, y)-i|}{4}\right]
$$




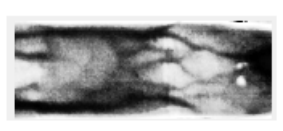

(a)

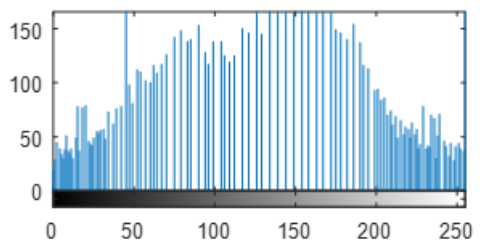

(b)

Figure 9.(a)After Fuzzy Histogram equalization, (b)Image Histogram

\subsubsection{Median filtering}

Median filtering is a non-linear process. To remove the impulsive and salt-and-pepper noise, median filtering is done on the image. For each pixel, a window is used to select neighboring pixels. For the pixels within this window, the median intensity value is computed and that median value is assigned to the pixel on which the operation was being performed. For each pixel of the image, this process is repeated [33]. It smoothly removes noise and preserves the edge-information. Strength of median filter depends upon window size [34]. For the experiments of this research, we used window size of $3 \times 3$ (see figure 10).

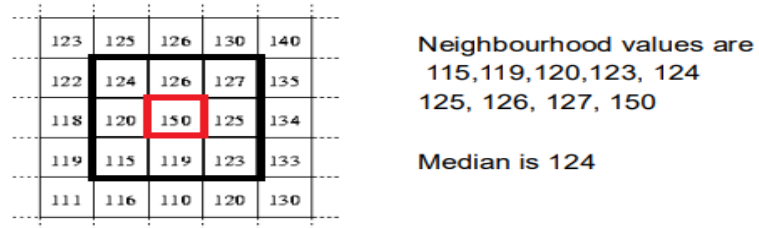

Figure 10. median filtering using $3 \times 3$ window size [18]

It soothes out the irregularities within texture as shown in the image (see figure 11):

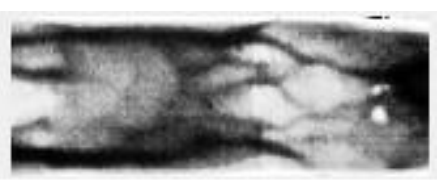

(a)

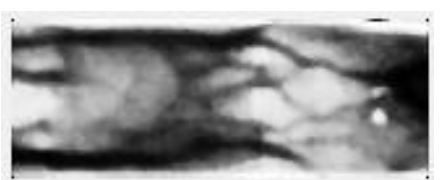

(b)

Figure 11.(a) Before median filtering,(b) After median filtering

\subsubsection{Gamma correction (power law) again}

To improve contrast and visibility, power law is again applied. Power law transform is represented by the equation [35]:

$$
S=C R^{\gamma}
$$

Here $\mathrm{C}$ and $\gamma$ are positive constants where $\gamma$ can be a value less or greater than 1 but a positive value. When value of $\mathrm{C}=1$, if value of $\gamma<1$ then the input image is changed into a new image. The intensities of pixels in this new image has a wide range in such a way that the image is much clear and bright and if the value of $\gamma>1$ then image is changed when it maps to intensities of pixels that have less range and so the image becomes dark [36].

In our experiments, we kept $\mathrm{C}=1$ and $\gamma=0.3$. In this technique, power law curves has fractional values of $\gamma$. These values are mapped to the median- filtered finger vein image (which had a darker range) to a transformed image (which had a broader range of pixels).

Final image corresponding to the last images(see figure 12). 


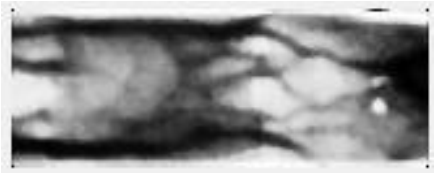

(a)

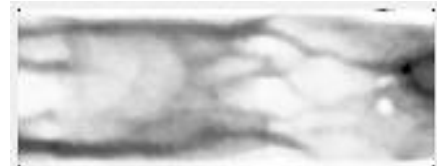

(b)

Figure 12 (a) Before Power law transform, (b)After Power law

Up till here we have discussed a method to bring out the unique shape of each finger vein pattern. We will call it Proposed method 1. We also carried out experimentation with changed orders of these enhancement steps and computed results. It was found that a rearrangement of these steps yielded slightly blurred images, but there was a distinct pattern in those images for each class and such images were giving marginally improved results as compared to Proposed method 1. We will call it Proposed method 2. A comparison of both these approaches is given in table 1, for person 103 (middle finger of left hand):

Table 1.Acomparison of two approaches

\begin{tabular}{|l|l|}
$\begin{array}{l}\text { Proposed method 1 with Cumulative histogram and } \\
\text { Fuzy histogram equalization } \\
\text { (CHFHE) }\end{array}$ & $\begin{array}{l}\text { Proposed method 2 with Fuzzy histogram } \\
\text { equalization } \\
\text { (FHE) }\end{array}$ \\
\hline Finger detection & Finger detection \\
\hline Gamma correction & Fuzzy histogram equalization \\
\hline Vein sharpening & \\
\hline Cumulative histogram equalization & Gamma correction \\
\hline & \\
\hline Fuzzy histogram equalization & Median filtering \\
\hline
\end{tabular}

\section{Feature extraction}

\subsection{HOG Feature extraction}

In tough visual situation, Histogram of Oriented Gradient (HOG) feature extraction is extensively used due to its ability to do well in those situations [37]. 
It extracts the information about target edge distribution and is a useful tool for shape identification [38] [39] [40] [41]. Shift and rotations of object do not affect this low-level feature descriptor [42] [43].

Its algorithm works as follows:

i. Image is separated into small sections which are called "cells".

ii. Manifestations of edge alignments in neighboring local section [44] are calculated for each cell (for 'signed gradients', histogram channels are spread evenly over 0- 360 and for 'unsigned' over 0-180).

iii. For scaling histogram counts, lighting effect is fixed. To do so, add energy measure for each local histogram over the larger connected sections.

iv. Feature vector, in the end, is accumulated by seeing all orientation counts in the calculated dominant direction of gradients.

The above stated steps therefore normalize the block- cells whose joint histograms establish the HOG feature vector of the complete image.

Scaling and rotation do not affect the algorithm because only the key points are used for scale-space extrema detection [44].

Following image(see figure 13) shows the gradient directions for a sample image patch:

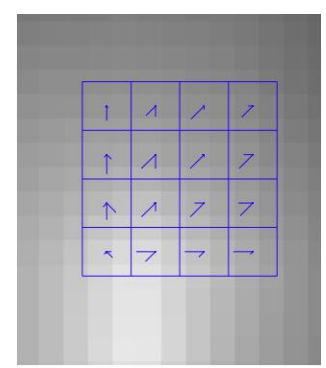

Figure 13. Sample HOG descriptors. Each cell of the patch shows the orientation of the gradients.

Thus, HOG descriptor primarily comprises of features about histograms of slopes of object edges of an image. HOG feature descriptor can be defined by the equation [45]:

$$
V_{H O G}=\left[F_{1}, F_{2}, F_{3}, \ldots \ldots \ldots \ldots, F_{k}\right]
$$

Where:

$\mathrm{K}=$ the total number of overlapping blocks in an image

$F_{i}$ is the normalized block vector of $i$-th block:

Where:

$$
F_{i}=\left[h_{1, i}, h_{2, i}, h_{3, i}, \ldots, h_{C * B, i}\right]
$$

$\mathrm{C}=$ total number of cells in a block

$\mathrm{B}=$ total number of bins in a cell

Bins create a cell and cells make up a block.

For a sample image of size $32 \times 32$, this procedure can be demonstrated (see figure 14) [45]:

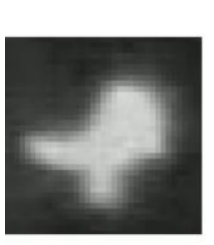

(a)

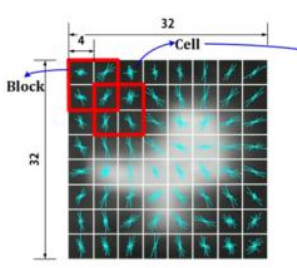

(b)

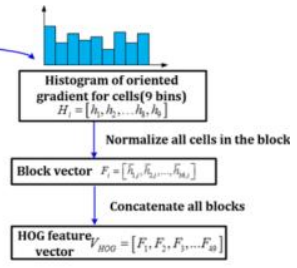

(c)

Figure 14. (a) Sample image (b) Visualization of gradients. Each block (red outline) contains four cells (white outline) (c) Process of generating HOG feature vector [45] 


\subsection{Hierarchical Centroid Shape Descriptor (HCSD)}

HCSD [46] [47] is fixed in kd-tree procedure decomposition [48]. It is a feature descriptor mainly for shapes. Centroid coordinates extracted from a binary image create this feature descriptor. It works by iteratively disintegrating an image into sub blocks of pixels by means of the kd-tree algorithm.

It comprises of data regarding centroid coordinates of local sections [49]. If $d$ denotes the depth of the features extraction procedure, then the length of this descriptor is specified by $2 \times(2 d-2)$.

Assume $I$ is the $M \times N$ binary image. Its foreground is denoted by Ifg and background by Ibg. HCSD is then created with the following stages [48]:

i. $\quad$ For input $I$, find its transpose: $I^{T}$,

ii. Find centroid $C(x c, y c)$ at the root level for every individual input:

Where:

$$
\begin{aligned}
& x_{c}=\frac{m_{10}}{m_{00}} \\
& y_{c}=\frac{m_{01}}{m_{00}}
\end{aligned}
$$

$m 10=$ first order moment along the $\mathrm{x}$-axis

$m 01=$ the first order moment along the y-axis

$m 00=$ area of $I f g$

Raw moment (also recognized as moment of order $(p+q)$ ) for a 2D continuous function $f(x, y)$ is specified by:

$$
m_{p q}=\int_{-\infty}^{\infty} \int_{-\infty}^{\infty} x^{p} y^{q} f(x, y) d x d y
$$

The raw moments $m_{p q}$ of a digital image with pixel intensities $I(i, j)$ are specified by:

$$
m_{p q}=\sum_{i=0}^{M} \sum_{j=0}^{N} i^{p} j^{q} I(i, j)
$$

iii. According to their centers of gravity $(\mathrm{x}=\mathrm{xc}, \mathrm{y}=\mathrm{yc})$ are Iteratively decomposed the image into two sub images until desired depth of breakdown is achieved. At each next stage, it is needed to change the axis of coordinates secured.

iv. As next step, attained vector is needed to scale over a range of -0.5 to 0.5 . Here, 0 is for the root-level centroid. Negative range represents tree decomposition of left sides and positive range represents right side.

v. Lastly, extracted features of the images I and $\mathrm{I}^{\mathrm{T}}$ are combined.

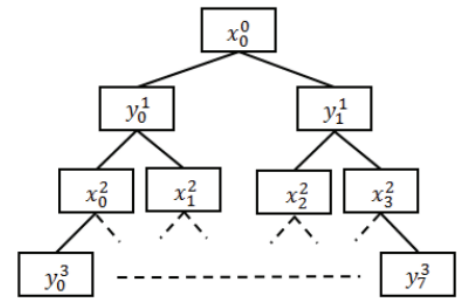

Figure 15. K-tree decomposition structure [48].

\section{Classification}

\subsection{K nearest neighbors}

$\mathrm{K}$ - nearest neighbor classifier, also called KNN [50] is a primitive but immensely favored classification method. The label of K closest samples is assigned to a test feature vector [51] [52].

It is a non- parametric passive algorithm [53]:

It is termed "non- parametric" as it does not cause any inherent assumptions about the placement of data into classes. 
It is termed "passive" because it does not utilize training data for generalization. It forecasts test data based on all the training data.

\subsection{Deep Neural Networks}

The process of learning data representations with many levels of abstraction through a set of computational models based on numerous processing layers is called "Deep Learning"(see figure 16) [54] [55]

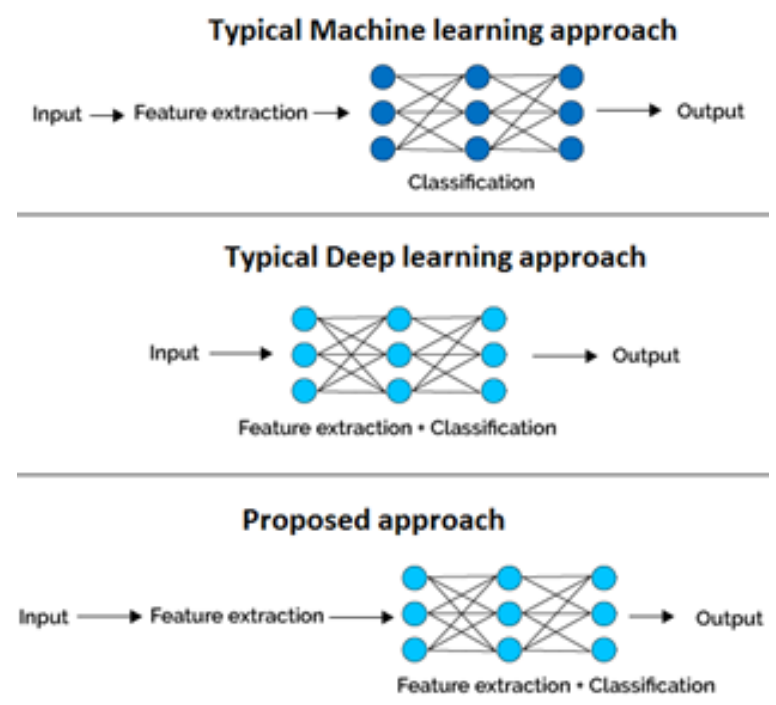

Figure 16.Deep Neural Network

\section{Results}

The computational time for each stage of the two proposed enhancement methods is presented in table 2 and 3

Table 2. Average computational time per step for method 1

\begin{tabular}{|l|l|}
\hline $\begin{array}{l}\text { Preprocessing Stages } \\
\text { of Proposed method 1 }\end{array}$ & $\begin{array}{l}\text { Average time taken } \\
\text { (seconds) }\end{array}$ \\
\hline Finger detection & 0.0072 \\
\hline Gamma correction & 0.0011 \\
\hline Image sharpening & 0.0036 \\
\hline $\begin{array}{l}\text { Cumulative Histogram } \\
\text { Equalization }\end{array}$ & 0.0028 \\
\hline $\begin{array}{l}\text { Fuzzy Histogram } \\
\text { Equalization }\end{array}$ & 0.0031 \\
\hline Median filtering & 0.0066 \\
\hline Power Law transform & 0.0014 \\
\hline Total time & 0.0258 \\
\hline
\end{tabular}

Table 3 . Average computational time per step for method 2

\begin{tabular}{|l|l|}
\hline $\begin{array}{l}\text { Preprocessing Stages } \\
\text { of Proposed method 2 }\end{array}$ & $\begin{array}{l}\text { Average time taken } \\
\text { (seconds) }\end{array}$ \\
\hline Finger detection & 0.0072 \\
\hline $\begin{array}{l}\text { Fuzzy histogram } \\
\text { equalization }\end{array}$ & 0.0031 \\
\hline Gamma correction & 0.0011 \\
\hline Median filtering & 0.0066 \\
\hline Weiner filtering & 0.0081 \\
\hline Gamma correction & 0.0011 \\
\hline Total time & 0.0272 \\
\hline
\end{tabular}

An extensive evaluation of the algorithm was performed. Every possible combination of finger type and hand type was tested for each feature extraction method and also for each classifier. It was found that the middle finger is the most discriminative of all for the considered dataset of 106 persons.

For the experimental results mentioned below, each finger of each hand is considered a separate category, even if it is of the same person(see figure 17). 


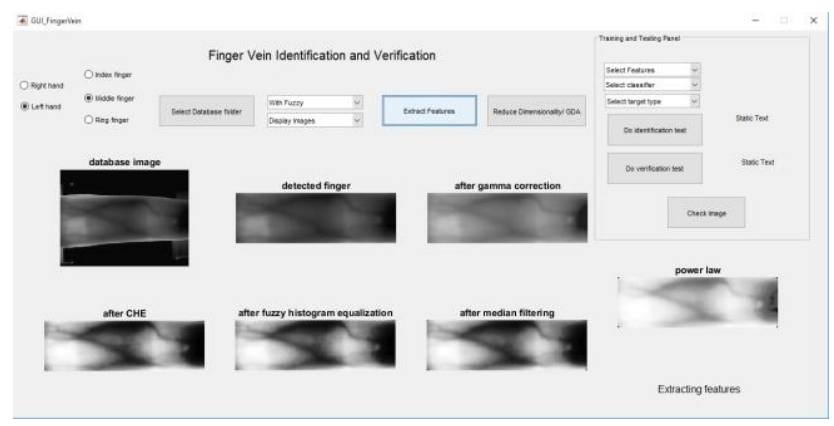

Figure 17.GUI for finger recognition and verification

Results of recognition and verification for middle finger of right hand, left hand and both hands are summarized in the tables below. For KNN, results are given with HOG, HC and with combined feature vector of HOG and HC. For DNN, dimensions of combined HOG and HC were first reduced (to be equal to 75) using Generalized Discriminant Analysis (GDA) [56] before giving them for classification. Reason for this is that data samples per class were limited and DNN is known to work best on extensive data whose feature length is not more than the total available samples(see tables $4,5,6,7,8,9)$.

Table 4. Results of KNN with right hand, middle

\begin{tabular}{|l|l|l|}
\hline Method & $\begin{array}{l}\text { Accuracy } \\
(\%)\end{array}$ & EER (\%) \\
\hline $\begin{array}{l}\text { Proposed upproach using } \\
\text { FHE with HOG }\end{array}$ & 99.0566 & 0.0089 \\
\hline $\begin{array}{l}\text { Proposed } \\
\text { approach using } \\
\text { FHE with HC }\end{array}$ & 99.0566 & 0.0089 \\
\hline $\begin{array}{l}\text { Proposed using } \\
\text { approach ush } \\
\text { FHE with } \\
\text { HOG+HC }\end{array}$ & 99.0566 & 0.0089 \\
\hline $\begin{array}{l}\text { Proposed using } \\
\text { approach } \\
\text { CHFHE with } \\
\text { HOG }\end{array}$ & 98.1132 & \\
\hline $\begin{array}{l}\text { Proposed using } \\
\text { approach usith } \\
\text { CHFHE with } \\
\text { HC }\end{array}$ & 98.1132 & 0.0178 \\
\hline $\begin{array}{l}\text { Proposed upproach using } \\
\text { CHFHE with } \\
\text { HOG+HC }\end{array}$ & \\
\hline
\end{tabular}

Table 5. Results of DNN with right hand, middle

\begin{tabular}{|c|c|c|}
\hline \multicolumn{3}{|c|}{ finger } \\
\hline Method & $\begin{array}{l}\text { Accuracy } \\
(\%)\end{array}$ & $\operatorname{EER}(\%)$ \\
\hline $\begin{array}{ll}\text { Proposed } & \\
\text { approach using } \\
\text { FHE with } \\
\text { dimension } \\
\text { reduced HOG + } \\
\text { HC }\end{array}$ & 89.6226 & 0.93895 \\
\hline $\begin{array}{l}\text { Proposed } \\
\text { approach using } \\
\text { CHFHE with } \\
\text { dimension } \\
\text { reduced HOG + } \\
\text { HC }\end{array}$ & 82.0755 & 0.09256 \\
\hline
\end{tabular}


Table 6. Results of KNN with left hand, middle finger

\begin{tabular}{|l|l|l|}
\hline Method & $\begin{array}{l}\text { Accuracy } \\
(\mathbf{\%})\end{array}$ & EER (\%) \\
\hline $\begin{array}{l}\text { Proposed upproach using } \\
\text { FHE with HOG }\end{array}$ & 97.1698 & 0.0267 \\
\hline $\begin{array}{l}\text { Proposed } \\
\text { approach using } \\
\text { FHE with HC }\end{array}$ & 93.3962 & 0.0623 \\
\hline $\begin{array}{l}\text { Proposed using } \\
\text { approach wHE } \\
\text { FHE with } \\
\text { HOG+HC with }\end{array}$ & 97.1698 & 0.0267 \\
\hline $\begin{array}{l}\text { Proposed upproach using } \\
\text { CHFHE with } \\
\text { HOG }\end{array}$ & 96.2264 & 0.0356 \\
\hline $\begin{array}{l}\text { Proposed upproach using } \\
\text { CHFHE with }\end{array}$ & 94.3396 & 0.0534 \\
HC & \\
\hline $\begin{array}{l}\text { Proposed upproach using } \\
\text { CHFHE with }\end{array}$ & 96.2264 & 0.0356 \\
HOG+HC & \\
\hline
\end{tabular}

Table 8. Results of KNN with both right and left hand, middle finger

\begin{tabular}{|c|c|c|}
\hline Method & $\begin{array}{l}\text { Accuracy } \\
(\%)\end{array}$ & EER (\%) \\
\hline $\begin{array}{l}\text { Proposed } \\
\text { approach using } \\
\text { FHE with HOG }\end{array}$ & 97.1698 & 0.014833 \\
\hline $\begin{array}{l}\text { Proposed } \\
\text { approach using } \\
\text { FHE with HC }\end{array}$ & 95.7547 & 0.019283 \\
\hline $\begin{array}{lr}\text { Proposed } & \\
\text { approach } & \text { using } \\
\text { FHE } & \text { with } \\
\text { HOG+HC } & \end{array}$ & 97.1698 & 0.014833 \\
\hline $\begin{array}{lr}\text { Proposed } & \\
\text { approach } & \text { using } \\
\text { CHFHE } & \text { with } \\
\text { HOG } & \end{array}$ & 96.6981 & 0.0178 \\
\hline $\begin{array}{lr}\text { Proposed } & \\
\text { approach } & \text { using } \\
\text { CHFHE } & \text { with } \\
\text { HC } & \end{array}$ & 95.7547 & 0.023733 \\
\hline $\begin{array}{lr}\text { Proposed } & \\
\text { approach } & \text { using } \\
\text { CHFHE } & \text { with } \\
\text { HOG+HC } & \end{array}$ & 96.6981 & 0.0178 \\
\hline
\end{tabular}

Table 7. Results of DNN with left hand, middle finger

\begin{tabular}{|c|c|c|}
\hline Method & $\begin{array}{l}\text { Accuracy } \\
(\%)\end{array}$ & $\operatorname{EER}(\%)$ \\
\hline $\begin{array}{l}\text { Proposed } \\
\text { approach using } \\
\text { FHE with } \\
\text { dimension } \\
\text { reduced HOG + } \\
\text { HC }\end{array}$ & 91.5094 & 0.93005 \\
\hline $\begin{array}{l}\text { Proposed } \\
\text { approach using } \\
\text { CHFHE with } \\
\text { dimension } \\
\text { reduced HOG + } \\
\text { HC }\end{array}$ & 89.6226 & 0.0969 \\
\hline
\end{tabular}

Table 9. Results of DNN with both right and left hand, middle finger

\begin{tabular}{|l|l|l|}
\hline Method & $\begin{array}{l}\text { Accuracy } \\
(\boldsymbol{\%})\end{array}$ & EER (\%) \\
\hline $\begin{array}{l}\text { Proposed approach using } \\
\text { FHE with }\end{array}$ & 91.5094 & 0.93005 \\
dimension & & \\
reduced HOG + & & \\
HC & & \\
\hline $\begin{array}{l}\text { Proposed approach using } \\
\text { CHFHE with } \\
\text { dimension } \\
\text { reduced HOG }+\end{array}$ & & \\
HC & & \\
\hline
\end{tabular}


From the above results, it can be concluded that KNN outperforms DNN by quite a wide margin. Reason for this can be attributed to the fact that DNN is much more suited for problems that have more extensive data. We also performed analysis of the proposed method using all fingers of both hands (636 category classification) present in the database for comparison with some state of the art recent benchmarks in this field.

Table 10. Results with all fingers of both hands with stratified 6 fold cross validation

\begin{tabular}{|c|c|c|c|}
\hline Reference & Method & $\begin{array}{l}\text { Accuracy } \\
(\%)\end{array}$ & $\begin{array}{l}\text { EER } \\
(\%)\end{array}$ \\
\hline [57] & $\begin{array}{ll}\text { Tri-Branch } & \text { Vein } \\
\text { Structure } & \\
\text { Analysis } & \\
\end{array}$ & - & 3.46 \\
\hline [16] & $\begin{array}{l}\text { Anatomy } \\
\text { Structure } \\
\text { Analysis }\end{array}$ & - & 1.39 \\
\hline \multirow[t]{3}{*}{ [14] } & $\begin{array}{l}\text { Deformation } \\
\text { information }\end{array}$ & 94 & 0.0268 \\
\hline & $\begin{array}{l}\text { Proposed } \\
\text { approach using } \\
\text { CHFHE with } \\
\text { HOG+HC+KNN }\end{array}$ & 95.8857 & 0.0123 \\
\hline & $\begin{array}{lr}\text { Proposed } \\
\text { approach using } \\
\text { FHE r with } \\
\text { HOG+HC+KNN }\end{array}$ & 97.6415 & 0.0037 \\
\hline
\end{tabular}

\section{Conclusion and future work}

In this paper, we introduce two novel methods of finger vein enhancement for recognition and verification purposes. An extensive evaluation of results is done with KNN and also with DNN. KNN proved to be much more accurate in its predictions on test data. Using stratified 6- fold analysis on all fingers of all hands in the SDUMLA database, a maximum accuracy of $97.6415 \%$ was achieved with an EER of 0.0037 . based on the analysis of the 106 persons present in the dataset, it was found that the most discriminative finger is the middle finger of right hand which gives above $99 \%$ accuracy.

For future work, the parameters of DNN can be optimized using some optimization methods [58] Furthermore, in this research we fed to DNN, feature vectors after reducing their dimensions to 75 using GDA. In future, analysis can be done by varying the length of this reduced dimensionality and also by experimenting with other dimension reduction techniques like Principal Component Analysis (PCA) [59]. Furthermore, Convolutional Neural Networks [60] can also be used to check performance using transfer learning techniques.

\section{References:}

[1] K. Shaheed, H. Liu, G. Yang, I. Qureshi, J. Gou and Y. Yin, "A Systematic Review of Finger Vein Recognition Techniques," MDPI, 2018.

[2] A. R. A. A. N. K. Jain, Introduction to Biometrics, Springer, 2011.

[3] K. N. Mishra, N. C. Mishra and A. Agrawal, "Veins Based Personal Identification Systems: A Review," I.J. Intelligent Systems and Applications, 2016.

[4] H. Qin, X. He, X. Yao and H. Li, "Finger-vein verification based on the curvature in Radon space," Expert Systems with Applications, 2017.

[5] A. R. S. K. Syazana-Itqan, N. M. Saad, N. A. Hamid and W. H. B. M. Saad, "A Review of FingerVein Biometrics Identification Approaches," Indian Journal Of Science and Technology, 2016.

[6] Y. Lu, S. Wu, Z. Fang, N. Xiong, S. Y. Dong and S. Park, "Exploring finger vein based personal 
authentication for secure IoT," Future Generation Computer Systems, 2017.

[7]M. \&. U. H. \&. U. S. Kono, "A new method for the identification of individuals by using of vein pattern matching of a finger," in Proceedings of the 5th Symposium on Pattern Measurement, 2000.

[8] F. Tagkalakis, D. Vlachakis, V. Megalooikonomou and A. Skodras, "A Novel Approach to Finger Vein Authentication," in 2017 IEEE 14th International Symposium on Biomedical Imaging (ISBI), 2017.

[9] X. Xi, L. Yang and Y. Yin, "Learning discriminative binary codes for finger vein recognition," Pattern Recognition, 2017.

[10] J. Yang and Y. S. G. Jia, "Finger-vein image matching based on adaptive curve transformation," Pattern Recognition, 2017.

[11] N. Miura, A. Nagasaka and T. Miyatake, "Feature extraction of finger-vein patterns based on repeated line tracking and its application to personal identification," Machine Vision and Applications, 2004.

[12] H. Zheng, Q. Xu, Y. Ye and W. Li, "Effects of meteorological factors on finger vein recognition," in 2017 IEEE International Conference on Identity, Security and Behavior Analysis (ISBA), 2017.

[13] H. Ding, "Anti-Spoofing a Finger Vascular Recognition Device with Pulse Detection," 2016.

[14] X. M. X. Y. Yin, "Science China Information Sciences," Finger vein recognition based on deformation information, 2018.

[15] Y. Yin and L. L. X. Sun, "SDUMLA-HMT: A Multimodal Biometric Database," in Chinese Conference on Biometric Recognition, 2011.

[16] G. Y. Y. Y. a. X. X. Lu Yang, "Finger Vein Recognition with Anatomy Structure Analysis," IEEE TRANSACTIONS ON CIRCUITS AND SYSTEMS FOR VIDEO TECHNOLOGY, 2017.

[17] Y. Yang, G. Yang and S. Wang, "Finger Vein Recognition Based on Multi-instance," International Journal of Digital Content Technology and its Applications, 2012.

[18] J. M. S. a. K. R. P. Wan Kim, "Multimodal Biometric Recognition Based on Convolutional Neural Network by the Fusion of Finger-Vein and Finger Shape Using Near-Infrared (NIR) Camera Sensor," Sensors MDPI, 2018.

[19] K. Akintoye, M. S. M. Rahim and A. H. Abdullah, "ENHANCEMENT OF FINGER VEIN IMAGE USING MULTIFILTERING ALGORITHM," ARPN Journal of Engineering and Applied Sciences, 2018.

[20] H. C. L. K. R. P. Eui Chul Lee, "Finger Vein Recognition Using Minutia-Based Alignment and Local Binary Pattern-Based Feature Extraction".

[21] K.-R. Park, Y.-K. Jang and B.-J. Kang, "A study on touchless finger vein recognition robust to the alignment and rotation of finger," The KIPS Transactions:PartB, 2008.

[22] S. A. Amiri and H. Hassanpour, "A Preprocessing Approach For Image Analysis Using Gamma Correction," International Journal of Computer Applications , 2012.

[23] K. Somasundaram and P. Kalavathi, "MEDICAL IMAGE CONTRAST ENHANCEMENT BASED ON GAMMA CORRECTION," International Journal of Knowledge Management \& e-Learning, 2011.

[24] C. W. ,. S. L. ,. M. R. ,. T. R. ,. N. K. ,. H. S. ,. Y.-H. Y. T. W. G. Jianga, "Image contrast 
enhancement with brightness preservation using an optimal gamma correction and weighted sum approach," Journal of Modern Optics, 2015.

[25]H. Hassanpour, N. Samadiani and S. Salehi, "Using morphological transforms to enhance the contrast of medical images," The Egyptian Journal of Radiology and Nuclear Medicine, 2015.

[26] B.Suresh, T. Kumari, P.Yashwanth and R. Rao., "Modified Histogram Based Contrast Enhancement Using Unsharp masking Filter For Medical Images," International Journal of Research in Computer and Communication Technology, 2015.

[27] T. Xiurong, "The application of adaptive unsharp mask algorithm in medical image enhancement," in Proceedings of 2011 Cross Strait Quad-Regional Radio Science and Wireless Technology Conference, 2011.

[28] S. Kansal, S. Purwar and R. K. Tripathi, "Image contrast enhancement using unsharp masking and histogram equalization," Multimedia Tools and Applications, 2018.

[29] R. J. C. M. J. H. P. F. T. D. M. Spring K, "Unsharp mask filtering. In: Interactive tutorials-optical microscopy primer," 2016.

[30] P. Garg and T. Jain, "A Comparative Study on Histogram Equalization and Cumulative Histogram Equalization," International Journal of New Technology and Research (IJNTR), 2017.

[31] "Simplified Teaching and Understanding of Histogram Equalization in Digital Image Processing," American Society for Engineering Education, 2009.

[32] D. Sheet, H. Garud, A. Suveer, M. Mahadevappa and J. Chatterjee, "Brightness Preserving Dynamic Fuzzy Histogram Equalization," IEEE Transactions on Consumer Electronics, 2010.

[33] M. Nagu and N. Shanker, "Image De-Noising By Using Median Filter and Weiner Filter,"

International Journal of Innovative Research in Computer and Communication Engineering, 2014.

[34] A. Makandar and B. Halalli, "Breast Cancer Image Enhancement using Median Filter and CLAHE," International Journal of Scientific \& Engineering Research, 2015.

[35] P. Janani, J. Premaladha and K. S. Ravichandran, "Image Enhancement Techniques: A Study," Indian Journal of Science and Technology, 2015.

[36] M.A.Ramiz and P. R. Quazi, "HYBRID TECHNIQUE FOR IMAGE ENHANCEMENT," International Research Journal of Engineering and Technology, 2017.

[37] S. A. Korkmaz, A. Akçiçek, H. Bínol and M. F. Korkmaz, "Recognition of the stomach cancer images with probabilistic HOG feature vector histograms by using HOG features," in 2017 IEEE 15th International Symposium on Intelligent Systems and Informatics (SISY), 2017.

[38] J. Ouyang, "Combining Extreme Learning Machine, RF and HOG for Feature Extraction," in IEEE Third International Conference on Multimedia Big Data (BigMM), 2017.

[39] S. A. Korkmaz, A. Akçiçek, H. Bínol and M. F. Korkmaz, "Recognition of the stomach cancer images with probabilistic HOG feature vector histograms by using HOG features," in IEEE 15th International Symposium on Intelligent Systems and Informatics (SISY), 2017.

[40] J. Chen, D. Zhou, Y. Wang, H. Fu and M. Wang, "Image feature extraction based on HOG and its application to fault diagnosis for rotating machinery," Journal of Intelligent \& Fuzzy Systems, 2018.

[41] C. Q. Lai and S. S. Teoh, "Efficiency Improvement in the Extraction of Histogram Oriented Gradient Feature for Human Detection Using Selective Histogram Bins and PCA," in 9th International Conference on 
Robotic, Vision, Signal Processing and Power Applications , 2016.

[42] A. Vashaee, R. Jafari, D. Ziou and M. Mehdi Rashidi, "Rotation Invariant HOG for Object Localization in Web Images," Signal Processing, 2016.

[43] C. Hang, F. Hu, A. E. Hassanien and K. Xiao, "Texture-based rotation-invariant Histograms of Oriented Gradients," in 11th International Computer Engineering Conference (ICENCO), 2016.

[44] O. Deniz, G. Bueno, J. Salido and F. D. 1. Torre, "Face recognition using Histograms of Oriented Gradients," Pattern Recognition Letters, 2011.

[45] Y. Ma, X. Wu, G. Yu, Y. Xu and Y. Wang, "Pedestrian Detection and Tracking from Low-Resolution Unmanned Aerial Vehicle Thermal Imagery," Sensors, 2016.

[46] S. Armon, "Handwriting Recognition and Fast Retrieval for Hebrew Historical Manuscripts," Hebrew University of Jerusalem, 2011.

[47] A. T. K. W. A. Sexton, "Font recognition using shape-based quad-tree and kd-tree decomposition," in Proceedings Of The Joint Conference On Information Sciences, 2000.

[48] E. Ilunga-Mbuyamba, J. G. Avina-Cervantes, D. Lindner and J. Guerrero-Turrubiate, "Automatic brain tumor tissue detection based on hierarchical centroid shape descriptor in Tl-weighted MR images," in 2016 International Conference on Electronics, Communications and Computers (CONIELECOMP), 2016.

[49] L. K. J. H. K.-H. J. Wahyono, "Similarity-based classification of 2-d shape using centroidbased tree-structured descriptor," Modern Advances in Applied Intelligence, Springer International Publishing, 2014.

[50] S. Zhang, X. Li, M. Zong, X. Zhu and R. Wang, "Efficient kNN Classification With Different Numbers of Nearest Neighbors," IEEE Transactions on Neural Networks and Learning Systems , 2018.

[51] A. Z. A. Zainuddin, W. Mansor, L. Y. Khuan and Z. Mahmoodin, "Classification of EEG Signal from Capable Dyslexic and Normal Children Using KNN," American Scientific Publishers, 2018.

[52] M. S. Sarma, Y. Srinivas, M. Abhiram, L. Ullala and M. S. Prasanthi, "Insider Threat Detection with Face Recognition and KNN User Classification," in IEEE International Conference on Cloud Computing in Emerging Markets (CCEM), 2017.

[53] R. S. P. S.Ponmani, "Classification Algorithms in Data Mining - A Survey," International Journal of Advanced Research in Computer Engineering \& Technology (IJARCET), 2017.

[54] L. B. R. F. L. T. M. P. Du Tran, "Learning Spatiotemporal Features with 3D Convolutional Networks," Computer Vision and Pattern Recognition, 2014.

[55] W. Liu, W. Li, L. Sun, L. Zhang and P. Chen, "Finger vein recognition based on deep learning," in 2017 12th IEEE Conference on Industrial Electronics and Applications (ICIEA), 2017.

[56] F. Bahmaninezhad and J. H. Hansen, "Generalized Discriminant Analysis (GDA) for Improved i-Vector Based Speaker Recognition," INTERSPEECH, 2016.

[57] L. Yang, G. Yang, X. Xi, X. Meng, C. Zhang and Y. Yin, "Tri-Branch Vein Structure Assisted Finger Vein Recognition," IEEE Access , vol. 5, 2017.

[58] P. Sun, W. Feng, R. Han, S. Yan and Y. Wen, "Optimizing Network Performance for Distributed DNN Training on GPU Clusters: ImageNet/AlexNet Training in 1.5 Minutes," Distributed, Parallel, and Cluster Computing, 2019.

[59] Y. Aït-Sahalia and D. Xiu, "Principal Component Analysis of High-Frequency Data," Journal of the American Statistical Association, 2017.

[60] M. Jamal, A. Arun, RossaErik and M.Shapirob, "On automated source selection for transfer learning in convolutional neural networks," Pattern recognition, 2018. 